\title{
The design of adaptive no-uniform filter banks
}

\author{
Liu Weidong Zhang Shen \\ China University of Mining and Technology \\ School of Information and Electric Engineering \\ Xuzhou, China ,221008 \\ lwdcumt@163.com,mengyuan_liu@163.com
}

\begin{abstract}
This paper proposed an efficient filter bank structure for adaptive noise cancellation algorithm, which is non-uniform sub-band decomposition algorithm based on the reference signal . In order to consider the signal approximation reconstruction, the design has adopted a prototype filter additional pre-emphasis filter method, and adopt uniform sub-band decomposition algorithm to carry out the idea of non-uniform sub-band decomposition. The algorithm is simple and easy to achieve, at the same time, as a result of sub-band decomposition depends on the distribution of the noise characteristics, so certain sub-band does not require the use of filtering methods, which not only significantly reduce the computation, but also improve the performance of adaptive filter convergence.

Keywords-protype filter, uniform filter,no-uniform filter
\end{abstract}

\section{INTRODUCTION}

Multi-rate filter banks have been a subject of considerable interest in the field of digital signal processing for many years $[1,6]$. One typical application of filter banks is in sub-band adaptive filtering, which has received much attention in recent years especially in the context of acoustic noise cancellation (ANC) ${ }^{[2,3]}$.In a typical sub-band filtering scheme for ANC, both the input (Acoustic emission) and reference (noise)signals are split into sub-bands by analysis banks. Adaptive filters are applied in each sub-band at a decimated rate and the sub-band residuals are recombined by a synthesis bank to create a fullband output signal at the original rate. Compared with the full-band case, sub-band adaptive filtering achieves a gain in computational complexity as a result of sampling rate reduction of the sub-band signals. However, due to aliasing effects in the analysis bank, critically sampled (i.e .maximally decimated) filter banks, which have received considerable attention in the literature and are used in sub-band coding, cannot be applied directly in a sub-band system for ANC ${ }^{[7,9]}$. Accordingly an oversampling scheme (i.e. non-critical sampling) is often used in sub-band to keep aliasing distortion in the full-band output of the synthesis bank below an acceptable level ${ }^{[8]}$. Thus, only amplitude and phase distortions need to be taken into account in the PR filter bank design, which leads to a relatively simpler design problem than in the critically sampled case. In ANC application, only a near perfect-reconstruction property is required for the filter bank, so that the design constraints are further relaxed.

The Major State Basic Research Development Program of China (2007CB209407))

\section{UNIFORM FILTER BANKS}

\section{A Design of Prototype Filters}

The filter bank design reduces to an appropriate choice of the prototype filter, which has two fulfill two criteria. Firstly the stopband attenuation is sufficiently large. A second constraint on the design is the perfect reconstruction condition in power complementary ${ }^{[4]}$ :

$$
\sum_{k=0}^{K-1}\left|H_{k}\left(e^{j \omega}\right)\right|^{2}=1
$$

Prototype design is to build complementary analysis and synthesis filter-banks, so that the reconstructed output signal contains very low distortion. The bandwidth of the synthesis filter $\pi / M$ is determined by the number of sub-bands $\mathrm{M}$ whereas the analysis filter bandwidth is larger and only limited by the decimation rate L. Our analysis and synthesis prototype filers are selected as in Figure 1.

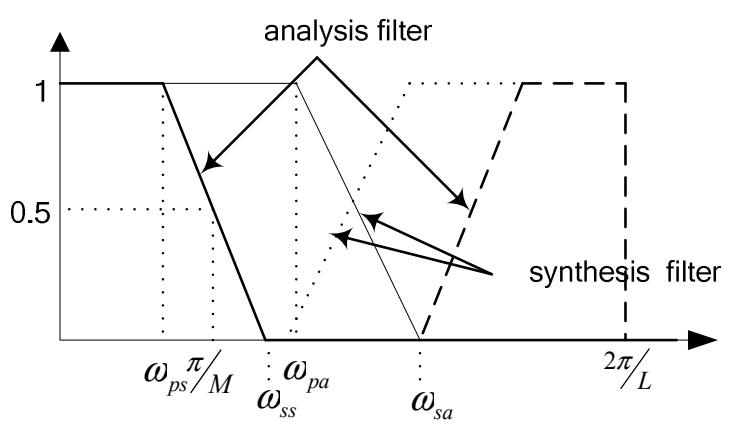

Figure 1. Analysis and Synthesis prototype filters

\section{B Pre-emphasis filter}

The oversampled input signals received by the subband processing blocks are no longer white in spectrum [4]. In fact, for oversampling factor of 3 , its bandwidth will be limited to $\mathrm{pi} / 3$. As a result, one would expect a slow convergence rate due to eigenvalue spread problem. On the other hand, while the oversampled subband signals are not white, their spectra are colored in a predicable way and can therefore be modified by fixed filters to "whiten" them in order to increase the convergence rate. Thus, the inherent benefit of decreased spectral dynamics resulting from subband decomposition is not lost due to oversampling.

Figure 2 shows a simplified representation of the subband spectra corresponding to white noise input into the filterbank, for a 3-times oversampled configuration. The dashed line 
shows the spectrum without pre-emphasis. As shown, nearly all the signal power is in the lower quarter of the spectrum. The signal power present in the upper three quarters of the spectrum is decided by the frequency response of the filterbank's prototype lowpass analysis filter.

We employ a pre-emphasis filter for each subband to amplify the low-level signal components in the high three quarters of the spectrum to flatten the spectrum, thereby reducing the signal's autocorrelation matrix eigenvalue spread, and increasing convergence rate. Figure 3 shows the frequency response of a typical pre-emphasis filter employed in the system.

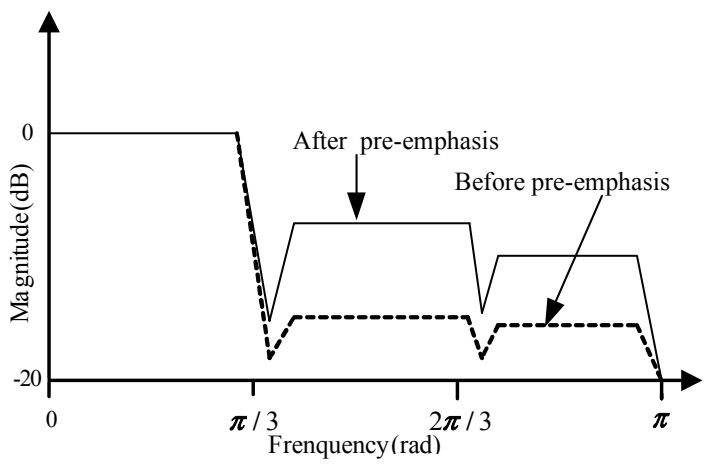

Figure 2. Simplified subband spectrum

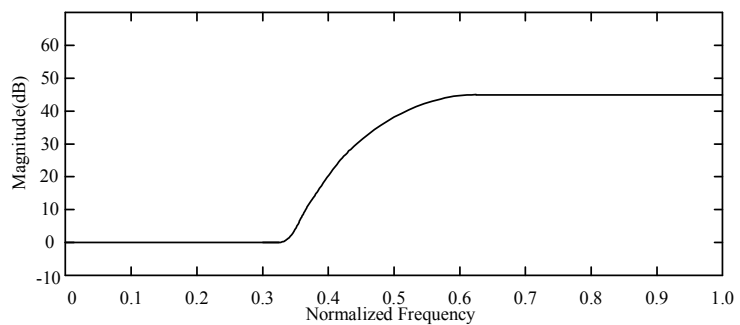

Figure 3. Pre-emphasis filter response

\section{NO-UNIFORM FILTER BANKS}

Sub-band decomposition in the Sub-band adaptive signal processing signals often overlooked signal characteristics of the noise considerations, mainly adopted uniform sub-band decomposition algorithm. In practice, the noise is often not distributed in the entire frequency range, in other words, some sub-band does not contain or contains weak noise signal, of these sub-band adaptive filtering, virtually an increase of unnecessary computation. In this paper, we take full advantage of the signal noise characteristics of acoustic emission signals in sub-band decomposition and take according to the signal noise characteristics, for non-uniform sub-band decomposition method.

\section{A Power Spectral Density}

If window on the sequence $x(n)$ to DFT analysis, such

$$
X(k)=\frac{1}{\sqrt{N_{d}}} \sum_{n=1}^{N_{d}} x(n) e^{-j \frac{2 \pi}{N_{d}} k(n-1)}
$$

Then the Power Spectral Density [5] is

$$
P_{x, x}[k]=\frac{2}{N_{w} f_{s}} \sum_{l=1}^{N_{w}}\left|\frac{1}{\sqrt{N_{d}}} \sum_{n=1}^{N_{d}} w(n) x^{l}(n) e^{-j \frac{2 \pi}{N_{d}} k(n-1)}\right|^{2}
$$

Where $N_{d}$ is the length of window, $N_{w}$ is the length of overlap data in every $N_{d}, f_{s}$ is the sample frequency, $w(n)$ is a window with

$$
\frac{1}{N_{d}} \sum_{n=1}^{N_{d}}|w(n)|^{2}=1
$$

$P_{x, x}[k]$ is the sequence $x(n)$ in frequency $f=k f_{s} /\left(N_{d}\right)$ 's Power Spectral Density.

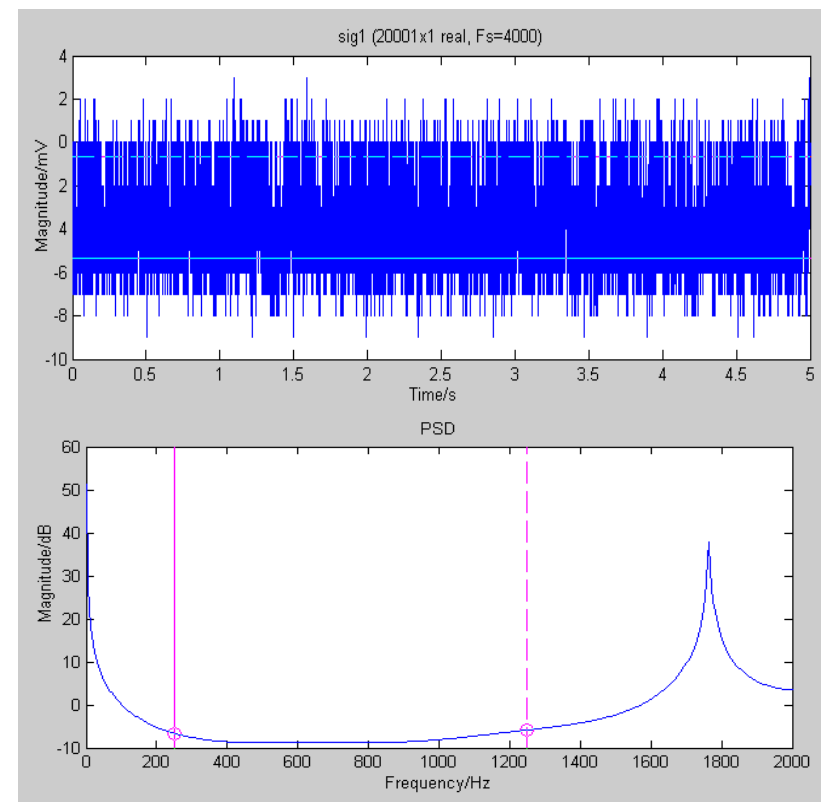

Figure 4. Acoustic emission signal and reference noise and their respective power spectral density

Through Figure 4 shows, the noise signal mainly distributed in frequency $[0 \sim \pi / 8]$ and $[5 \pi / 8 \sim \pi]$, so we only adopted adaptive filtering in these sub-bands, but sub-band $[\pi / 8 \sim 5 \pi / 8]$ didn't need to filter because it not contains noise signal .

\section{B Non-uniform sub-band adaptive decomposition algorithm}

Carrying out power spectrum Density analysis to the reference noise signal and in accordance with the noise distribution to perform adaptive non-uniform sub-band decomposition, the specific algorithm is as follows:

1) Design uniform filter banks with $\mathrm{K}$ sub-bands $l=\left\{l_{1}, l_{2}, \cdots l_{K}\right\}$; 
2) Based PSD of the noise signal, estimate the subband's energy $\hat{\mathcal{E}}=\left\{\varepsilon_{1}, \varepsilon_{2}, \cdots \mathcal{E}_{K}\right\}$ and the all sub-bands average energy $\bar{\varepsilon}=\frac{1}{K} \sum_{i=1}^{K} \varepsilon_{i}$;

3) Perform sub-band adaptive non-uniform partition to the input signal, find $\{j\}$ in $\hat{\varepsilon}=\left\{\varepsilon_{1}, \varepsilon_{2}, \cdots \mathcal{E}_{K}\right\}$, suppose the initial value $j=1, m=1$; Calculate $\left|\hat{\varepsilon}_{i}-\bar{\varepsilon}\right|$ and According to the actual noise signal to determine the threshold $s t d \varepsilon$ value. Now suppose the first sub-band's energy meet the conditions $\left|\hat{\varepsilon}_{1}-\bar{\varepsilon}\right|>s t d \varepsilon$; the Specific algorithm as shown in Figure 5 .

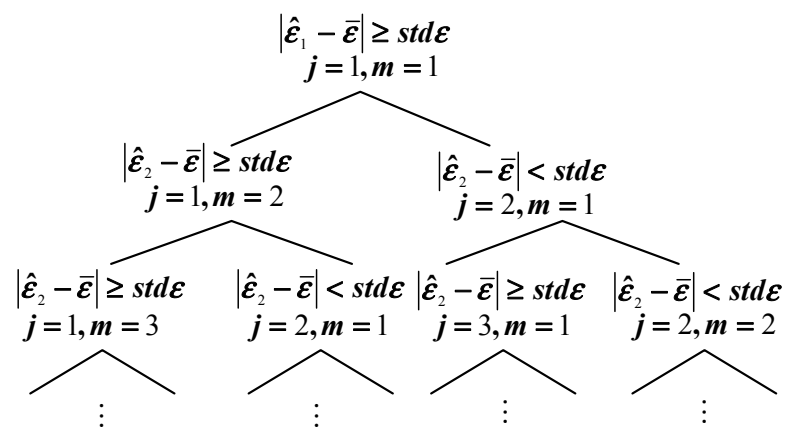

Figure 5. Non-uniform sub-band decomposition algorithm

According to Figure 5 shows the algorithm, we can determine the number of non-uniform sub-bands, and each sub-band's bandwidth. Assuming a total of 10 uniform sub-band in Figure 6 in a variety of colors of the thin solid line . the non-uniform sub-band decomposition algorithm proposed in this paper is adopted,we can get $j=1, m=2$;

$j=2, m=5 j=3, m=3$, That is to say, the signal is divided the three non-uniform sub-band decomposition as shows in Figure 6 in the thick solid line.

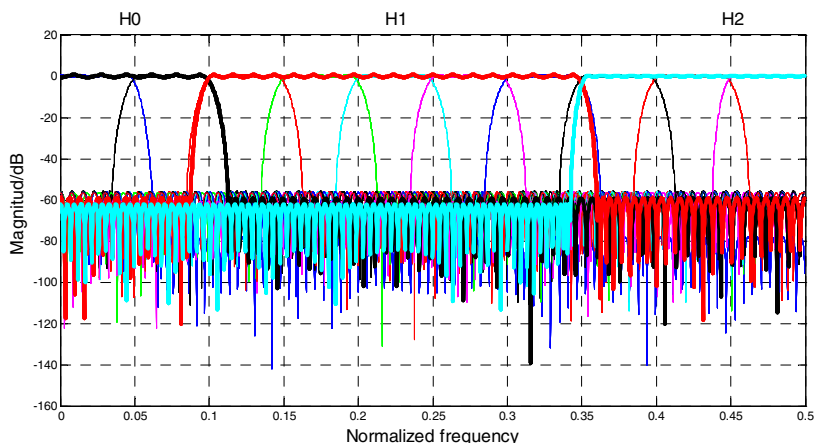

Figure 6. ten sub-band uniform filter banks and three non-uniform sub-band filter banks

We present a number of simulations using the subband adaptive fileter. The impulse response used in the simulations has length 1600 and closely resembles that of simulation signal. The normalised LMS algorithm is used as the individual subband adaptive filters with step chosen at 0.5 , the full-band LMS filter is used as a comparison. Simulation resulms shows the performance of the No-uniform subband adaptive filter over the fullband and the uniform subband adative .

\section{CONCLUSIONS}

This paper presents a filter bank model of adaptive no-uniform subband decomposition, which breakthroughs the idea of uniform subband decomposition in the past, gives more consideration of the signal to noise distribution characteristics in the adaptive noise cancellation, The simulation shows that the adaptive non-uniform subband adaptive filter compared with full-band adaptive filters and uniform sub-band filter, both the calculation of the reduction and its convergence rate has a lot of improvement. This algorithm provides a new way of thinking and direction in the sub-band adaptive filter with the subband decomposition.

\section{ACKNOWLEDGMENT}

This work was supported by grants from the Major State Basic Research Development Program of China (2007CB209407) and the National High Technology Research(2006AA06Z119).

\section{REFERENCE}

[1] Departement Elektrotechniek ESAT-SISTA/TR 1997-23;A Relation Between Subband and Frequency-Domain Adaptive Filtering; Koen Eneman, Marc Moonen

[2] Subband adaptive filtering with real-valued subband signals for acoustic echo cancellation;W. H.Chin and B. Farhang-Boroujeny;IEE Proc.-Vis. Image Signal Process., Vol. 148, No. 4, August 2001;

[3]Realization of an adaptive algorithm with subband filtering approach for acoustic echo cancellation in telecommunication applications; malte sandrock,stefan schmitt;DSPecialists GmbH,D-10245 Berlin,Germany 2001

[4] Tam, Hamid Sheikhzadeh, Todd Schneider. Highly oversampled subband adaptive filters for noise for cancelation on a low-resource DSP system. King

[5] Recommendation for an algorithm for Power Spectral Density estimation for LISA Pathfinder;S. Vitale, G. Heinzel, K. Danzmann, W. Weber, M. Hueller, M. Hewitson;September 22,2006;

[6]Digital Signal Processing Principles, Algorithms and Applications (Third Edition) [America] John G.Proakis Dimitris G.manolakis 2004

[7] Haykin, S. Adaptive Filter Theory. Prentice Hall, Upper Saddle River, 3rd Edition, 1996.

[8] L IU Weidong, DING Enjie. The Research of Prediction Technology of Rock Burst, Indust ry and Mine Automation, P18-20,No. 5 ,Oct . 2006

[9] ECCTD'01 - European Conference on Circuit Theory and Design, August 28-31, 2001, Espoo, Finland;Signal Adaptive Subband Decomposition for Adaptive Noise Cancellation; Riitta Niemisto and Ioan Tabu 